\title{
Limiting magnitude for Dome C optical interferometers
}

\author{
T. Elhalkouj ${ }^{1}$, R. G. Petrov ${ }^{2}$, M. Lazrek $^{1}$ \\ and Z. Benkhaldoun ${ }^{1}$ \\ ${ }^{1}$ Université Cady-Ayyad, Faculté des sciences, LPHEA, Marrakech, Maroc \\ ${ }^{2}$ Université de Nice Sophia-Antipolis, UMR 6525, Parc Valrose, 06108 Nice, France
}

\begin{abstract}
This paper discusses the potential performances of interferometers at the Dome $\mathrm{C}$ site in the Antarctic. The work is focussed on the limiting performances for fringe tracking, considering different fringe trackers architectures. The fringe tracking limiting magnitude is combined with estimates of the isopistonic angle at Dome $\mathrm{C}$ to evaluate the possibilities of off axis fringe tracking. The first evaluations presented here indicate that the sky coverage with realistic off axis fringe tracking is quite high at Dome $\mathrm{C}$ even with modest individual apertures and this could finally the best and decisive advantage of this site for optical interferometry. This might make a Dome C interferometer an unique deep sky very high angular resolution machine.
\end{abstract}

Keywords. instrumentation: interferometers, atmospheric effects, site testing.

\section{Introduction}

The limiting magnitude of a ground based multi aperture interferometer is set mainly by its capacity to cophase the apertures by actively correcting the differences of atmospheric piston. In many cases this cophasing cannot be performed on the science source itself and so far there is no artificial source really working solution. The solution which is being implemented for example on the VLTI is to use an off axis reference star. Given the current limiting magnitude for fringe tracking and the isopistonic angle at Paranal (defined by the angle within which two sources are supposed to be producing the same atmospheric piston) give a very poor sky coverage for the VLTI. We try to investigate what is the limiting magnitude for a large number of apertures and, after combining it with estimates of the isopistonic angle for different sites, we evaluate the conditions for a good sky coverage in the direction of the galactic pole.

\section{Limiting magnitude of cophasing}

We have considered two cophasing methods (described in figure 1). In the Global one, all fringes peaks in Fourier space are processed and the $N(N-1) / 2$ baselines are used to find the $N-1$ piston differences. In the Hierarchic approach we cophase pairs of telescope, then pairs of pairs and so on. The SNR is evaluated from the hight of the fringe peaks above the noise level in Fourier space [Petrov (1989)]. Using the fundamental noises (photon noise from the source photon flux $(\Gamma)$, thermic noise from the background $(\Lambda)$, detector readout noise $\left.\left(\sigma_{L}=10 e^{-} / \mathrm{pixel}\right)\right)$ and the number $N$ of telescopes, we derived the SNR for the Global and Hierarchical cophasing respectively in equations 2.1 and 2.2.

$$
(S N R)^{-2}=2 \frac{N-1}{N \Gamma}\left(1+\frac{\Lambda}{\Gamma}+\frac{16 N \sigma_{L}^{2}}{\Gamma}\right)+\frac{1}{4 N^{2}(N-1) \Gamma^{2}}\left[1+\frac{\Lambda}{\Gamma}+\frac{16 N \sigma_{L}^{2}}{\Gamma}\right]^{2}
$$




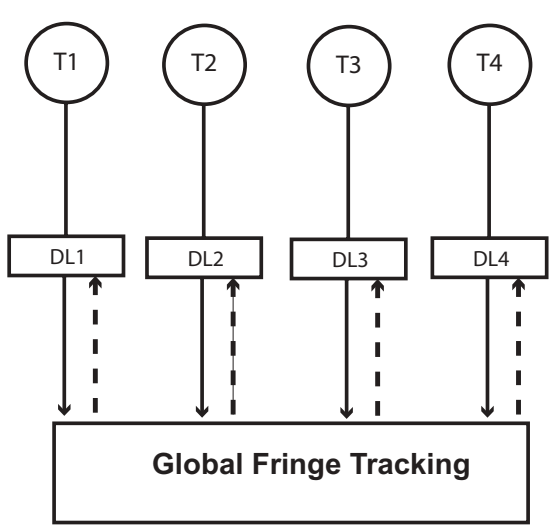

(a)

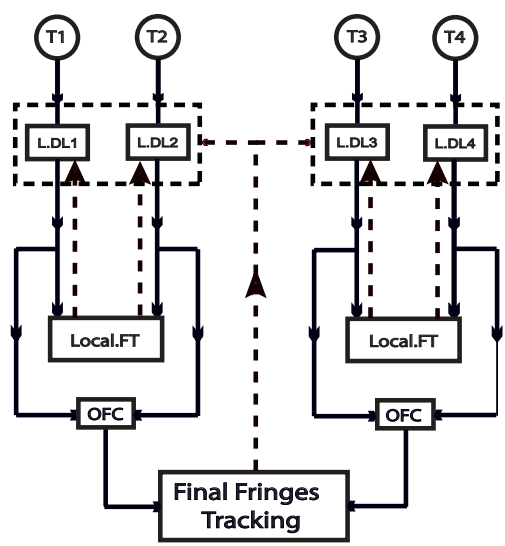

(b)

Figure 1. Global and hierarchical cophasing per pairs. DL: Delay Line; L.DL : Local DL; FT : Fringe Tracking; OFC: Optical Fiber Combinator.

$$
(S N R)^{-2}=\frac{\log _{2}(N)}{\Gamma}\left(1+\frac{\Lambda}{\Gamma}+\frac{32 \sigma_{L}^{2}}{\Gamma}\right)+\frac{\log _{2}(N)}{16 \Gamma}\left[1+\frac{\Lambda}{\Gamma}+\frac{32 \sigma_{L}^{2}}{\Gamma}\right]^{2}
$$

The background flux $\Lambda$ has been computed from a black body law with temperature $T$, the exposure time $\tau$ and the full $\mathrm{K}$ band. The instrument global efficiency was $2 \%$ and the emissivity $95 \%$. The results are given in figure 2 showing the limiting magnitudes at Paranal $(T=300 K)$ and Dome $\mathrm{C}(T=200 K)$. We note that the hierarchical cophasing is better, since the performance decrease much less with the telescope number. In the following, we restrain to that case.

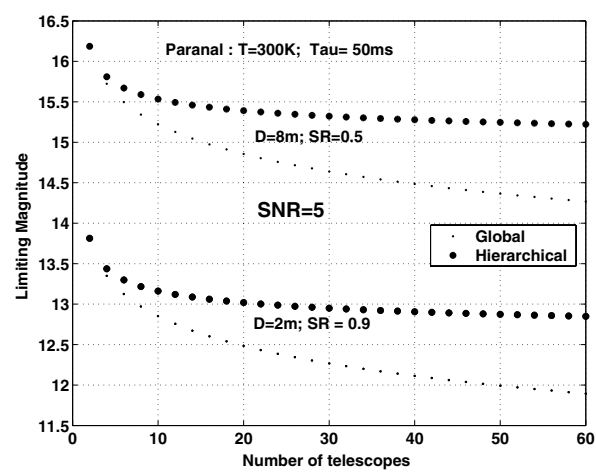

(a)

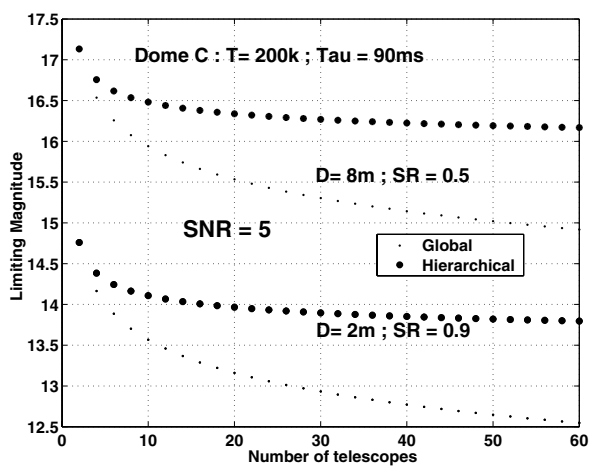

(b)

Figure 2. Limiting magnitude of cophasing for Paranal (a) and Dome C (b) for $8 \mathrm{~m}$ (Strehl ratio $\mathrm{SR}=0.5)$ and $2 \mathrm{~m}(\mathrm{SR}=0.9)$ telscopes.

\section{Comparing Dome $\mathrm{C}$ and Paranal}

\subsection{Limiting magnitude and sky coverage}

Figure 3 compares the limiting magnitude at Paranal and Dome $\mathrm{C}$ sites for an array of 8 meter telescopes. The gain in thermal background and incoherence time gives a clear 
advantage to the Dome C. It has been made by computing from equation 2.1 the flux, and therefore the magnitude yielding a $S N R=5$. Figure 4 shows the radius $R(50 \%)$ of the sky patch in which we have a probability of 0.5 to find a star brighter than a given limiting magnitude. If we have an estimate of the isopistonic angle for a site, we can find from figure 4 what is the cophasing limiting magnitude necessary to have a $50 \%$ to find a guide star in the isopistonic domain. This curve has been computed using CDS catalogs and counting the number of stars as a function of the diameter of a sky area. The sky direction have been produced randomly for values close to the galactic pole. For a given interferometer on a given site, the limiting magnitude corresponds to an individual aperture diameter.

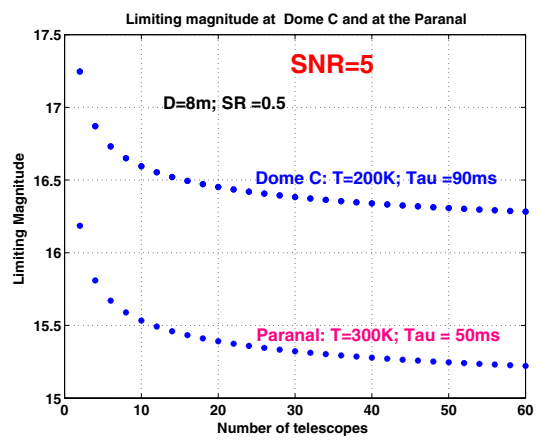

Figure 3. Limiting magnitude at Dome $\mathrm{C}$ and Paranal.

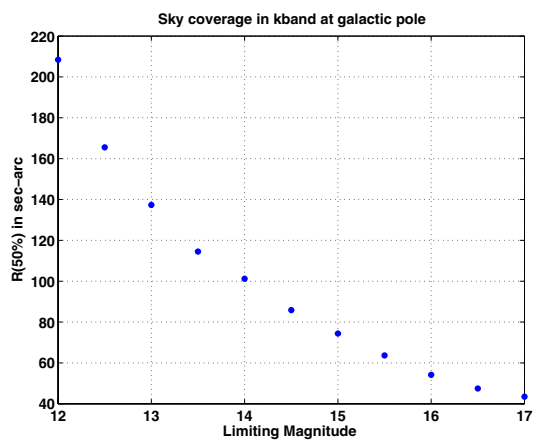

Figure 4. Sky coverage in the galactic pole.

\subsection{Isopistonic angle}

The isopistonic angle is the angle between two sources producing the same piston, with a $\lambda / S N R$ accuracy. It is deduced from the classical measured isoplanetic angle of the given site according to [Esposito \& al (2000)]. In this preliminary work, we have assumed that the ratio between the isopistonic and the isoplanetic angle is the same at Dome $\mathrm{C}$ and at Paranal. This basically assumes that the outer scale is the same, which is a conservative estimate. The demonstration showing that this isopistonic/isoplanetic ratio is dominated by the outer scale exceeds the possible length of this paper and is to be published soon. From [Esposito \& al (2000)] and the measured Paranal isoplanetic angle (1.9 arcsec in the visible and therefore 9.9 arcsec in $\mathrm{K}$ ) we found a Paranal isopistonic angle $=57$ arcsec in the $\mathrm{K}$ band. The most recent summer time measures made by [Aristidi (2005)] yield a Dome C median isoplanetic angle in the visible of 6.8 arcsec. Extrapolating [Esposito \& al (2000)] work therefore yields a Dome $C$ isopistonic angle $=121$ arcsec in the $\mathrm{K}$ band. This value should be quite correct for the winter Dome $\mathrm{C}$ conditions. It has been shown that the seeing is quite different in winter and in summer, but almost all the difference is produced within the first 30 meters above the ground, which has almost no impact on the isoplanetic nor isopistonic angles. Using figure 4, the isopistonic values at Dome $\mathrm{C}$ and Paranal give the cophasing limiting magnitude necessary to have a $50 \%$ sky coverage in the direction of the Galactic Pole:

$$
\begin{gathered}
\operatorname{mag}(\text { Galactic Pole, K band, Paranal })=16 \\
\operatorname{mag}(\text { Galactic Pole, K band, Dome } \mathrm{C})=13.2
\end{gathered}
$$

\section{3. individual aperture diameter}

Figure 5 displays the diameter of the individual aperture necessary to achieve the cophasing limiting magnitude needed for a $50 \%$ sky coverage near the galactic pole, for a $\lambda / 5$ 


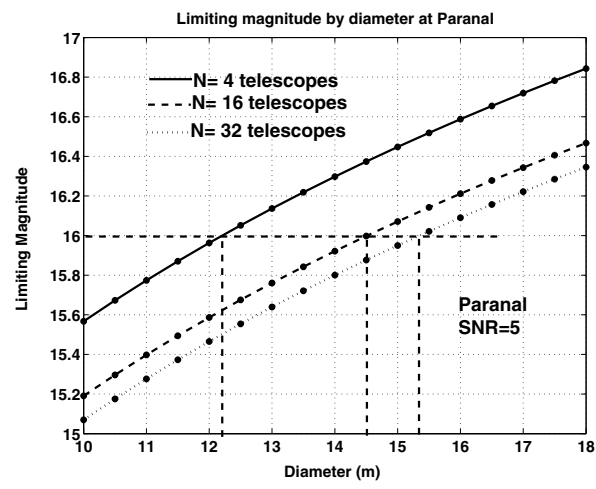

(a)

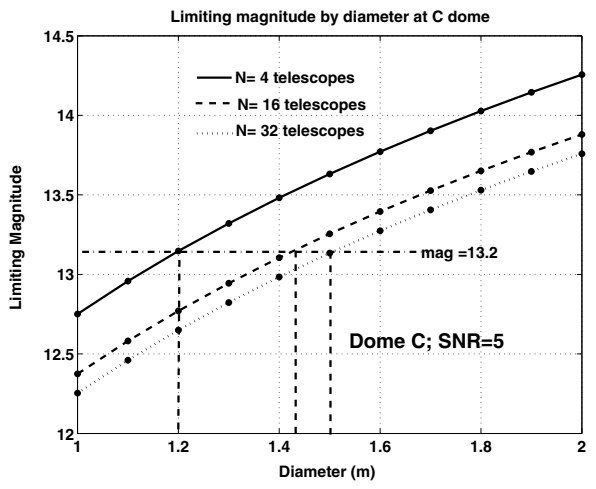

(b)

Figure 5. Limiting magnitude with the diameter at Paranal and at Dome C.

fringe tracking quality. At Paranal, the need to have $K=16$ yields and individual telescope size ranging from $12 \mathrm{~m}$ to $16 \mathrm{~m}$, depending on the number of telescopes of the array. Note that the above computation has been made for the seeing of the best $25 \%$ Paranal nights. At Dome $\mathrm{C}$, the needed magnitude is only $K=13.2$ for a median seeing and the corresponding individual aperture diameter goes from $1.2 \mathrm{~m}$ for 4 telescopes to $1.6 \mathrm{~m}$ for more than 32 apertures.

\section{Conclusion}

This preliminary study shows that a Dome C array with typically $1.5 \mathrm{~m}$ individual aperture is able to ensure a sky coverage better than $50 \%$ near the galactic pole. On a good classical site like Paranal a similar requirement implies using individual apertures in the $16 \mathrm{~m}$ class. Therefore, the Dome $\mathrm{C}$ might be the single place on earth were a diluted, and therefore very high angular resolution, aperture can achieve very high magnitudes. Indeed, since the fringe tracking is permitted by a straightforward use of off axis stars, the limiting magnitude for the science source will be set only by the total collecting surface of the interferometer. Of course, our study is preliminary. We have to demonstrate more accurately the relation between isopostonic values and measured seeing characteristics and eventually measure directly the in situ isopistonic angle. We have also to consider other parameters, such as the limiting conditions for the adaptive correction of the unit aperture, but at least in the near infrared this should be a minor problem for telescopes smaller than $2 \mathrm{~m}$. If further studies confirm this evaluation, a Dome $\mathrm{C}$ interferometer would be an absolutely unique possibility for very high angular resolution deep sky studies.

\section{Acknowledgements}

We sincerely thank Dr A. Ziad, for his efficient help concerning the isoplanetic angles, and the Data Center of Strasbourg (CDS,2MASS).

\section{References}

Esposito, S. \& Riccardi, A. \& Femenía 2000, A.\&A. Letter. to.Editor. 353. L29, L32 (2000) Aristidi, E. 2005, Private communication

Petrov, R.G. 1989, NATO Advanced Science Institutes (ASI) Series C, Volume 274, p. 249

Ziad, A. \& al 2000, Aplied. Optics. Vol.39. No.30. p. 5415 